\title{
Slowly rotating black hole solutions in Horndeski gravity
}

\author{
Andrea Maselli, ${ }^{1, *}$ Hector O. Silva, ${ }^{2, \dagger}$ Masato Minamitsuji, ${ }^{3, \neq}$ and Emanuele Berti ${ }^{2,3, \S}$ \\ ${ }^{1}$ Center for Relativistic Astrophysics, School of Physics, Georgia Institute of Technology, \\ Atlanta, Georgia 30332, USA \\ ${ }^{2}$ Department of Physics and Astronomy, The University of Mississippi, University, Mississippi 38677, USA \\ ${ }^{3}$ Departamento de Física, CENTRA, Instituto Superior Técnico, Universidade de Lisboa, \\ Avenida Rovisco Pais 1, 1049 Lisboa, Portugal
}

(Received 22 August 2015; published 25 November 2015)

\begin{abstract}
We study black hole solutions at first order in the Hartle-Thorne slow-rotation approximation in Horndeski gravity theories. We derive the equations of motion including also cases where the scalar depends linearly on time. In the Hartle-Thorne formalism, all first-order rotational corrections are described by a single frame-dragging function. We show that the frame-dragging function is exactly the same as in general relativity for all known black hole solutions in shift-symmetric Horndeski theories, with the exception of theories with a linear coupling to the Gauss-Bonnet invariant. Our results extend previous nohair theorems for a broad class of Horndeski gravity theories.
\end{abstract}

\section{INTRODUCTION}

General relativity (GR) has passed all experimental tests in the Solar System and in binary pulsars with flying colors [1]. Current observations mostly probe the weakfield/slow-motion regime of the theory (with the exception of binary pulsars, where the orbital motion is nonrelativistic but the individual binary members are compact objects), and some of the most interesting strong-field predictions of GR are still elusive and difficult to verify. Observational and theoretical issues with Einstein's theory-including the dark matter and dark energy problems, the origin of curvature singularities and the quest for an ultraviolet completion of GR-have motivated strong efforts to develop modified theories of gravity which differ from GR in the infrared and ultraviolet regimes, while being consistent with the stringent observational bounds at intermediate energies [2]. The search for unambiguous signatures of modifications of GR in the strong-gravity regime is a major goal of several research fields, including cosmology [3], "standard" electromagnetic astronomy [4], and Earthand space-based gravitational-wave astronomy [5,6].

In this work we consider a class of modifications of GR known as Horndeski gravity [7]. This is the most general scalar-tensor theory with a single scalar yielding secondorder field equations for the metric and the scalar field (see e.g. $[8,9]$ for tensor-multiscalar theories, and $[10,11]$ for multiscalar versions of Horndeski gravity). All the terms present in the action of Horndeski gravity have been shown to be originating from Galileons, i.e. scalar-tensor models having Galilean symmetry in flat space-time [12].

\footnotetext{
*andrea.maselli@roma1.infn.it

hosilva@phy.olemiss.edu

masato.minamitsuji@ist.utl.pt

§eberti@olemiss.edu
}

"Generalized Galileon" theories in curved space-time in any number of dimensions were studied in [13], and shown to be equivalent to Horndeski gravity in four dimensions in [14]. Furthermore, Horndeski gravity can be shown to emerge from a Kaluza-Klein compactification of higherdimensional Lovelock gravity (see e.g. [15] for an introduction to this topic, and for a discussion of the relation between exact solutions in Lovelock and Horndeski gravity).

The equations of motion of Horndeski gravity can be derived from the action

$$
S=\sum_{i=2}^{5} \int d^{4} x \sqrt{-g} \mathcal{L}_{i},
$$

where

$$
\begin{aligned}
\mathcal{L}_{2}= & G_{2}, \\
\mathcal{L}_{3}= & -G_{3} \square \phi, \\
\mathcal{L}_{4}= & G_{4} R+G_{4 \mathrm{X}}\left[(\square \phi)^{2}-\phi_{\mu \nu}^{2}\right], \\
\mathcal{L}_{5}= & G_{5} G_{\mu \nu} \phi^{\mu \nu} \\
& -\frac{G_{5 \mathrm{X}}}{6}\left[(\square \phi)^{3}+2 \phi_{\mu \nu}^{3}-3 \phi_{\mu \nu}^{2} \square \phi\right] .
\end{aligned}
$$

Here $g_{\mu \nu}$ is the metric tensor, $g \equiv \operatorname{det}\left(g_{\mu \nu}\right)$, and $R$ and $G_{\mu \nu}$ are the Ricci scalar and the Einstein tensor associated with $g_{\mu \nu}$, respectively. We have introduced the functions $G_{i}=G_{i}(\phi, X)$, which depend only on the scalar field $\phi$ and its kinetic energy $X=-\partial_{\mu} \phi \partial^{\mu} \phi / 2$, and we use units such that the reduced Planck mass $m_{\mathrm{Pl}}^{2}=(8 \pi G)^{-1}=1$. For brevity we have also defined the shorthand notation 
$\phi_{\mu \ldots \nu} \equiv \nabla_{\mu} \ldots \nabla_{\nu} \phi, \quad \phi_{\mu \nu}^{2} \equiv \phi_{\mu \nu} \phi^{\mu \nu}, \quad \phi_{\mu \nu}^{3} \equiv \phi_{\mu \nu} \phi^{\nu \alpha} \phi_{\alpha}^{\mu}$ and $\square \phi \equiv g^{\mu \nu} \phi_{\mu \nu}$. Horndeski theories are an interesting phenomenological playground for strong-field gravity because they include as special cases all dark energy and modified gravity models with a single scalar degree of freedom:

(1) the GR limit corresponds to $G_{4}(\phi, X)=1 / 2$, with $G_{2}=G_{3}=G_{5}=0$;

(2) when the only nonzero term is $G_{4}(\phi, X)=F(\phi)$ we recover a scalar-tensor theory with nonminimal coupling of the form $F(\phi) R$, and therefore BransDicke theory and $f(R)$ gravity are special cases of Horndeski gravity;

(3) Einstein-dilaton-Gauss-Bonnet (EdGB) gravity, i.e. a theory with action

$$
S=\int d^{4} x \sqrt{-g}\left(\frac{1}{2} R+X+\xi(\phi) R_{\mathrm{GB}}^{2}\right),
$$

where $R_{\mathrm{GB}}^{2}=R^{2}-4 R_{\mu \nu} R^{\mu \nu}+R_{\alpha \beta \gamma \delta} R^{\alpha \beta \gamma \delta}$ is the Gauss-Bonnet invariant, corresponds to setting

$$
\begin{aligned}
& G_{2}=X+8 \xi^{(4)} X^{2}(3-\ln X), \\
& G_{3}=4 \xi^{(3)} X(7-3 \ln X), \\
& G_{4}=\frac{1}{2}+4 \xi^{(2)} X(2-\ln X), \\
& G_{5}=-4 \xi^{(1)} \ln X,
\end{aligned}
$$

where $R_{\alpha \beta \gamma \delta}$ and $R_{\mu \nu}$ are the Riemann and Ricci tensors, and we have defined $\xi^{(n)} \equiv \partial^{n} \xi / \partial \phi^{n}$ [14];

(4) a theory with nonminimal derivative coupling of the form

$$
S=\int d^{4} x \sqrt{-g}\left[\zeta R+2 \eta X+\beta G^{\mu \nu} \phi_{\mu} \phi_{\nu}-2 \Lambda_{0}\right]
$$

(see e.g. [16-20] for cosmological studies of this type of action) corresponds to the following choice ${ }^{1}$ of the coupling functions [21]:

$$
\begin{aligned}
& G_{2}=-2 \Lambda_{0}+2 \eta X, \\
& G_{4}=\zeta+\beta X, \\
& G_{3}=G_{5}=0,
\end{aligned}
$$

where $\Lambda_{0}, \eta, \zeta$ and $\beta$ are constants;

\footnotetext{
${ }^{1}$ A coupling of the form $G^{\mu \nu} \phi_{\mu} \phi_{\nu}$ can also be obtained by setting $G_{5}=-\phi$ and integrating by parts.
}

(5) the Lagrangian $\mathcal{L}_{2}$ corresponds to the k-essence field [22-24] (and therefore part of the literature uses a different notation, where $G_{2}$ is denoted by $K$ );

(6) the covariant Galileon of Ref. [25] is recovered by setting $G_{2}=-c_{2} X, G_{3}=-c_{3} X / M^{3}, G_{4}=$ $M_{\mathrm{Pl}}^{2} / 2-c_{4} X^{2} / M^{6}$ and $G_{5}=3 c_{5} X^{2} / M^{9}$, where the $c_{i}(i=2, \ldots, 5)$ are constants and $M$ is a constant with dimensions of mass.

In this paper we are interested in black hole $(\mathrm{BH})$ solutions in Horndeski gravity. As one of the most striking strong-field predictions of GR, BHs are ideal astrophysical laboratories to test gravity in the strong-field regime. Various authors explored nonrotating $\mathrm{BH}$ solutions in special classes of Horndeski gravity. Rinaldi [26] studied $\mathrm{BH}$ solutions in theories with a nonminimal derivative coupling to the Einstein tensor of the form $G^{\mu \nu} \phi_{\mu} \phi_{\nu}$. Minamitsuji [27] and Anabalon et al. [28] found more general solutions by adding a cosmological constant. Kobayashi and Tanahashi [21] studied BH solutions in a subclass of Horndeski theories that is both shift-symmetric (i.e., symmetric under $\phi \rightarrow \phi+c$, with $c$ a constant) and reflection-symmetric (i.e., symmetric under $\phi \rightarrow-\phi$ ). Under these assumptions, the only nonzero terms in the action are $\mathcal{L}_{2}$ and $\mathcal{L}_{4}$. Theories with nonminimal derivative coupling are both shift- and reflection-symmetric, and therefore they are a subclass of the theories considered in Ref. [21].

The nonrotating $\mathrm{BH}$ solutions found in the works listed above either reduce to the Schwarzschild solution or are not asymptotically flat. This is a consequence of the no-hair theorem by Hui and Nicolis [29], which states that vacuum, static, spherically symmetric, asymptotically flat BHs have no hair in Horndeski theories with shift symmetry. As pointed out by Sotiriou and Zhou [30,31] the theorem actually has a loophole: asymptotically flat solutions can exist for theories of the EdGB type with $\xi(\phi)=\phi$ (these theories are still shift-symmetric, because the GaussBonnet combination is a topological invariant). The solutions found in Refs. [30,31] are effectively special cases of the nonrotating EdGB BH solutions studied by Kanti et al. [32], that were subsequently generalized to slow rotation in Refs. [33-35] and to rapid rotation in Refs. [36,37]. Other possibilities to violate the no-hair theorems include adding a time dependence to the scalar (but not to the metric), as in the solution proposed by Babichev and Charmousis [38], or considering biscalar extensions of Horndeski gravity [11]. Reference [39] extended the solutions in [38] to the charged case, allowing for a coupling of the derivative of the scalar field to the energy-momentum tensor of the Maxwell field. Reference [40] argued that a phase transition to charged hairy $\mathrm{BHs}$ can be realized through a nonminimal derivative coupling to the Einstein tensor; in this case, however, the equations of motion were solved perturbatively.

The key question we address in this paper is the following: does rotation produce interesting violations of the no-hair 
theorem at leading order in a slow-rotation expansion? In experimental terms, could we possibly observe violations of the no-hair theorem via frame-dragging experiments? The conclusion of our analysis is that frame-dragging corrections are exactly the same as in $G R$ for all of the Horndeski BH solutions that we analyzed, with the (already known) exception of BH solutions in EdGB gravity [30-35]. We do not expect this result to hold at second order in rotation, and this will be the topic of a follow-up study.

The plan of the paper is as follows. In Sec. II we present the equations of motion for slowly rotating $\mathrm{BH}$ space-times in Horndeski gravity, and we carry out some basic sanity checks (in particular, we check that GR and EdGB gravity are recovered in the appropriate limits). The field equations themselves are rather lengthy, and they are listed in
Appendix for the reader's convenience. In Sec. III we study slowly rotating BHs in theories with a nonminimal derivative coupling with the Einstein tensor, finding that frame-dragging corrections are exactly the same as in GR. In Sec. IV we provide arguments (based on the work of Refs. [29-31]) to support this no-hair result. Finally, in Sec. V we present some conclusions and point out directions for future work.

\section{THE EQUATIONS OF MOTION}

The equations of motion that follow from the action (1) can be written schematically as $\mathcal{E}_{\alpha \beta}=0$ (from variations of the metric) and $\mathcal{E}_{\phi}=0$ (from variations of the scalar field), where

$$
\begin{aligned}
\mathcal{E}_{\alpha \beta}= & -\frac{g_{\alpha \beta}}{2} G_{2}+G_{2 \mathrm{X}} X_{\alpha \beta}-\left[G_{3 \mathrm{X}} X_{\alpha \beta} \square \phi+\frac{1}{2} g_{\alpha \beta} G_{3 \mu} \phi^{\mu}-G_{3(\alpha} \phi_{\beta)}\right]+G_{\alpha \beta} G_{4}+G_{4 \mathrm{X}} X_{\alpha \beta} R+G_{4 \mu}{ }^{\mu} g_{\alpha \beta}-G_{4 \alpha \beta} \\
& +\left[G_{4 \mathrm{XX}} X_{\alpha \beta}-\frac{1}{2} G_{4 \mathrm{X}} g_{\alpha \beta}\right]\left(\square \phi^{2}-\phi_{\mu \nu}^{2}\right)+2 \square \phi G_{4 \mathrm{X}} \phi_{\alpha \beta}-2 \nabla_{(\alpha}\left[G_{4 \mathrm{X}} \phi_{\beta)} \square \phi\right]+\nabla_{\mu}\left[G_{4 \mathrm{X}} \phi^{\mu} \square \phi\right] g_{\alpha \beta} \\
& +2 \nabla_{\mu}\left[G_{4 \mathrm{X}} \phi_{(\alpha} \phi_{\beta)}^{\mu}\right]-\nabla_{\mu}\left[G_{4 \mathrm{X}} \phi^{\mu} \phi_{\alpha \beta}\right]-2 G_{4 \mathrm{X}} \phi_{\beta \nu} \phi_{\alpha}^{\nu}+G_{\mu \nu} \phi^{\mu \nu}\left(G_{5 \mathrm{X}} X_{\alpha \beta}-\frac{1}{2} G_{5} g_{\alpha \beta}\right)+2 G_{5} \phi^{\mu}{ }_{(\beta} G_{\alpha) \mu} \\
& -\nabla^{\mu}\left[G_{5} \phi_{(\alpha} G_{\beta) \mu}\right]+\frac{1}{2} \nabla^{\mu}\left[G_{5} \phi_{\mu} G_{\alpha \beta}\right]+\frac{1}{2}\left\{R G_{5} \phi_{\alpha \beta}-R_{\alpha \beta} G_{5} \phi_{\mu}^{\mu}+\square\left(G_{5} \phi_{\alpha \beta}\right)+\nabla_{\alpha} \nabla_{\beta}\left(G_{5} \phi_{\mu}{ }^{\mu}\right)-2 \nabla_{\mu} \nabla_{(\alpha}\left[G_{5} \phi_{\beta)}{ }^{\mu}\right]\right. \\
& \left.+g_{\alpha \beta}\left[\nabla_{\mu} \nabla_{\nu}\left(G_{5} \phi^{\mu \nu}\right)-\square\left(G_{5} \phi_{\nu}{ }^{\nu}\right)\right]\right\}-\frac{1}{6}\left(G_{5 \mathrm{XX}} X_{\alpha \beta}-\frac{1}{2} g_{\alpha \beta} G_{5 \mathrm{X}}\right)\left[(\square \phi)^{3}+2 \phi_{\mu \nu}^{3}-3 \phi_{\mu \nu}^{2} \square \phi\right] \\
& -\frac{1}{2}\left\{G_{5 \mathrm{X}}(\square \phi)^{2} \phi_{\alpha \beta}-2 \nabla_{(\alpha}\left[G_{5 \mathrm{X}}(\square \phi)^{2} \phi_{\beta)}\right]+\frac{1}{2} g_{\alpha \beta} \nabla^{\mu}\left[G_{5 \mathrm{X}}(\square \phi)^{2} \phi_{\mu}\right]\right\} \\
& -\left\{G_{5 \mathrm{X}} \phi_{\mu \alpha} \phi_{\beta \sigma} \phi^{\sigma \mu}-\nabla^{\sigma}\left[G_{5 \mathrm{X}} \phi_{(\alpha} \phi_{\mu \sigma} \phi_{\beta)}^{\mu}\right]+\frac{1}{2} \nabla^{\sigma}\left[G_{5 \mathrm{X}} \phi_{\sigma} \phi_{\mu \alpha} \phi_{\beta}^{\mu}{ }_{\beta}\right]\right\} \\
& +\frac{1}{2}\left\{G_{5 \mathrm{X}}\left(\phi_{\mu \nu}^{2} \phi_{\alpha \beta}+2 \square \phi \phi_{\alpha \mu} \phi_{\beta}^{\mu}\right)-\nabla_{(\beta}\left[G_{5 \mathrm{X}} \phi_{\alpha)} \phi_{\mu \sigma} \phi^{\mu \sigma}\right]+\frac{1}{2} g_{\alpha \beta} \nabla^{\sigma}\left[G_{5 \mathrm{X}} \phi_{\sigma} \phi_{\mu \nu} \phi^{\mu \nu}\right]-2 \nabla^{\mu}\left[G_{5 \mathrm{X}} \square \phi \phi_{(\alpha} \phi_{\beta) \mu}\right]\right. \\
& \left.+\nabla^{\mu}\left[G_{5 \mathrm{X}} \square \phi \phi_{\mu} \phi_{\alpha \beta}\right]\right\},
\end{aligned}
$$

$$
\begin{aligned}
\mathcal{E}_{\phi}= & G_{2 \phi}+\nabla_{\alpha}\left(G_{2 \mathrm{X}} \phi^{\alpha}\right)-G_{3 \alpha}{ }^{\alpha}-\nabla_{\alpha}\left(G_{3 \mathrm{X}} \phi^{\alpha} \square \phi\right)-\square \phi G_{3 \phi}+G_{4 \phi} R+\left(\square \phi^{2}-\phi_{\mu \nu}^{2}\right) G_{4 \mathrm{X} \phi} \\
& +\nabla^{\alpha}\left[G_{4 \mathrm{XX}} \phi_{\alpha}\left(\square \phi^{2}-\phi_{\mu \nu}^{2}\right)\right]+\nabla^{\alpha}\left(G_{4 \mathrm{X}} \phi_{\alpha} R\right)+2 \square\left(G_{4 \mathrm{X}} \square \phi\right)-2 \nabla^{\alpha} \nabla^{\beta}\left(G_{4 \mathrm{X}} \phi_{\alpha \beta}\right)+G_{5 \phi} G_{\alpha \beta} \phi^{\alpha \beta} \\
& +G_{5}^{\alpha \beta} G_{\alpha \beta}-\frac{1}{6} G_{5 \mathrm{X} \phi}\left[(\square \phi)^{3}+2 \phi_{\mu \nu}^{3}-3 \phi_{\mu \nu}^{2} \square \phi\right]+\nabla^{\alpha}\left[G_{5 \mathrm{X}} \phi_{\alpha} \phi_{\mu \nu} G^{\mu \nu}\right]-\frac{1}{6} \nabla^{\alpha}\left\{G _ { 5 \mathrm { XX } } \phi _ { \alpha } \left[(\square \phi)^{3}\right.\right. \\
& \left.\left.+2 \phi_{\mu \nu}^{3}-3 \phi_{\mu \nu}^{2} \square \phi\right]\right\}-\frac{1}{2} \square\left[G_{5 \mathrm{X}}(\square \phi)^{2}\right]-\nabla^{\alpha} \nabla^{\beta}\left[G_{5 \mathrm{X}} \phi_{\alpha}^{\mu}{ }_{\alpha} \phi_{\mu \beta}\right]+\frac{1}{2} \square\left(G_{5 \mathrm{X}} \phi_{\mu \nu}^{2}\right)+\nabla^{\alpha} \nabla^{\beta}\left(G_{5 \mathrm{X}} \phi_{\alpha \beta} \square \phi\right) .
\end{aligned}
$$

Here we have defined $G_{i \alpha} \equiv \nabla_{\alpha} G_{i}, X_{\alpha \beta} \equiv \delta X / \delta g_{\alpha \beta}$, and $f_{1(\alpha} f_{2 \beta)} \equiv\left(f_{1 \alpha} f_{2 \beta}+f_{1 \beta} f_{2 \alpha}\right) / 2$. These equations apparently contain higher derivatives, but they can be shown to be of second order using appropriate identities (cf. e.g. Appendix B of [14]).
To investigate the properties of slowly rotating $\mathrm{BH}$ solutions in Horndeski gravity we follow the approach developed by Hartle [41,42], in which rotational corrections to the static, spherically symmetric background are introduced within a perturbative framework. At linear order 
in the $\mathrm{BH}$ angular velocity $\Omega$, the metric can be written in the form

$$
\begin{aligned}
d s^{2}= & -A(r) d t^{2}+\frac{d r^{2}}{B(r)}+r^{2}\left(d \theta^{2}+\sin ^{2} \theta d \varphi^{2}\right) \\
& -2 \omega(r) d t d \varphi
\end{aligned}
$$

where the frame-dragging function $\omega(r)$ is of order $\Omega$.

Kobayashi et al. $[43,44]$ carried out a fully relativistic analysis of linear perturbations around static, nonrotating, spherically symmetric backgrounds. As a preliminary step for this perturbative analysis, they derived the equations of motion for general static, spherically symmetric vacuum space-times. Here we generalize these results to the slowly rotating case, deriving the equations of motion for the metric component $\omega(r)$. We also generalize the analysis of Refs. $[43,44]$ by allowing the scalar field to depend on the radial and time coordinates, since a nontrivial time dependence of $\phi$ allows for the existence of hairy BHs [38]. Following Refs. [21,38], we assume the scalar field to have the functional form

$$
\phi=\phi(t, r)=q t+\psi(r) .
$$

Then the kinetic energy $X$ is independent of $t$ :

$$
X=X(r)=\frac{1}{2}\left(\frac{q^{2}}{A(r)}-B(r) \psi^{\prime 2}\right)
$$

where the prime means differentiation with respect to the radial coordinate $r$. Then in (15), $X_{\alpha \beta}=$ $-\psi^{\prime 2} \delta_{\alpha}^{r} \delta_{\beta}^{r} / 2-q^{2} / 2 \delta_{\alpha}^{t} \delta^{t}{ }_{\beta}$. The $t t$ and $r r$ components of Eq. (15) yield two equations,

$$
\begin{aligned}
\mathcal{E}_{t t} & =0, \\
\mathcal{E}_{r r} & =0,
\end{aligned}
$$

and the scalar field equation of motion (16) in the background metric (17) is given by

$$
\mathcal{E}_{\phi}=0,
$$

where the explicit form of the left-hand sides of (20)-(22) is quite lengthy, and it can be found in the Appendix. For a static scalar field $(q=0)$, Eqs. (20)-(22) reproduce the results obtained in $[43,44]$; for reflection-symmetric theories, they reduce to the results of [21].

For slowly rotating solutions at linear order in the $\mathrm{BH}$ angular velocity, the only nonvanishing component of the equations of motion yields a second-order ordinary differential equation for the variable $\omega(r)$ :

$$
\mathcal{E}_{t \varphi}=0 \text {. }
$$

Again, the explicit form of the left-hand side can be found in the Appendix.

Taken together, Eqs. (20)-(23) provide a full description of vacuum space-times at linear order in rotation. We now consider two special cases as sanity checks of the equations of motion.

\section{A. General relativity}

As stated in the introduction, the Einstein-Hilbert Lagrangian of GR corresponds to setting $G_{4}=1 / 2$ and all the other functions equal to zero. In this case the equation of motion for the function $\omega(r)$ simply reads

$$
\omega^{\prime \prime}+\frac{\omega^{\prime}}{2}\left(\frac{B^{\prime}}{B}+\frac{8}{r}-\frac{A^{\prime}}{A}\right)=0,
$$

in agreement with the frame-dragging equation found by Hartle [41]. If the nonrotating background is the Schwarzschild solution this further simplifies to

$$
\omega^{\prime \prime}+\frac{4}{r} \omega^{\prime}=0 .
$$

\section{B. Einstein-dilaton-Gauss-Bonnet gravity}

EdGB gravity [32] corresponds to the choice of Eqs. (7)(10). If the coupling is linear in the field-i.e. $\xi(\phi)=\alpha \phi$ as in $[30,31]$, so that the theory is shift-symmetric-and $q=0$, we get

$$
\begin{gathered}
\left(8 \alpha B \phi^{\prime}-r\right) \omega^{\prime \prime}+\left[12 \alpha \phi^{\prime} B^{\prime}+8 \alpha B \phi^{\prime \prime}+\frac{24 \alpha}{r} B \phi^{\prime}\right. \\
\left.-4 \alpha B \phi^{\prime} \frac{A^{\prime}}{A}+\frac{r}{2}\left(\frac{A^{\prime}}{A}-\frac{B^{\prime}}{B}\right)-4\right] \omega^{\prime}=0 .
\end{gathered}
$$

If instead we use an exponential coupling of the form $\xi=e^{\phi}$ and we set $q=0$, the frame-dragging equation becomes

$$
\begin{aligned}
& \omega^{\prime \prime}\left(\frac{2}{B} r^{2}-2 r e^{\phi} \phi^{\prime}\right)+\frac{\omega^{\prime} r}{B(r)}\left(8-r \frac{A^{\prime}}{A}+\frac{B^{\prime}}{B} r\right) \\
& -\omega^{\prime} e^{\phi}\left[2 \phi^{\prime \prime} r+6 \phi^{\prime}+r \phi^{\prime}\left(3 \frac{B^{\prime}}{B}+2 \phi^{\prime}-\frac{A^{\prime}}{A}\right)\right]=0,
\end{aligned}
$$

in agreement with the result of Ref. [33].

\section{NONMINIMAL DERIVATIVE COUPLING TO THE EINSTEIN TENSOR}

In this section we apply the formalism derived above to rotating solutions in a class of Horndeski theories characterized by a nonminimal derivative coupling with the Einstein tensor of the form (11). The theory defined by this action is invariant under both shift symmetry $(\phi \rightarrow \phi+c)$ and reflection symmetry $(\phi \rightarrow-\phi)$. Shift 
symmetry allows us to write the equation of motion for the scalar field $\phi$ as a current conservation equation $[30,31,38]$ :

$$
\nabla_{\mu} J^{\mu}=0
$$

In particular, for the action (11), the conservation equation (28) reduces to

$$
\left(\eta g^{\mu \nu}-\beta G^{\mu \nu}\right) \nabla_{\mu} \partial_{\nu} \phi=0 .
$$

Moreover, following [21] we shall parametrize our solutions in terms of three auxiliary functions:

$$
\begin{aligned}
\Lambda & =-\frac{\eta}{\beta}, \\
\mathcal{F}(X) & =-\frac{-2 X \beta \eta+\zeta \eta+\beta \Lambda_{0}}{2 X \beta^{2}}, \\
\mathcal{G}(X) & =2(\zeta-\beta X) .
\end{aligned}
$$

Using this parametrization, $\mathrm{BH}$ configurations within this theory can be easily obtained with the following procedure. The $t t$ component of the equations of motion, Eq. (20), leads to the equation

$$
-\frac{2 A(r)^{2}}{q^{2} r \mathcal{G}} \frac{d}{d r}\left[X \mathcal{G}\left(1-r^{2} \mathcal{F}(X)\right)\right]=0,
$$

which can be integrated with the solution

$$
X \mathcal{G}^{2}(X)\left[1-r^{2} \mathcal{F}(X)\right]=C,
$$

where $C$ is a constant. Equation (34) determines $X(r)$ algebraically. Then the metric function $A(r)$ can be found by solving Eq. (21), which yields

$$
(r A)^{\prime}=\frac{q^{2}}{2 X} \frac{1-r^{2} \Lambda}{1-r^{2} \mathcal{F}(X)} .
$$

Finally, the metric function $B(r)$ can be found from Eq. (28):

$$
B(r)=\frac{2 X}{q^{2}}\left[1-r^{2} \mathcal{F}(X)\right] A(r) .
$$

With the choice (30), the frame-dragging equation for $\omega(r)$ has a particularly simple form:

$$
\mathcal{G} \omega^{\prime \prime}+\omega^{\prime}\left[\mathcal{G}_{\mathrm{X}} X^{\prime}+\frac{1}{2}\left(\frac{8}{r}-\frac{A^{\prime}}{A}+\frac{B^{\prime}}{B}\right) \mathcal{G}\right]=0 .
$$

As an extension of Ref. [21], we now consider nonrotating BH solutions of Eqs. (34)-(36) in different subcases and investigate the slow-rotation corrections predicted by Eq. (37) for each of these solutions.

Case 1: $\mathcal{F}=0$. One possibility to satisfy Eq. (34) is to impose $\mathcal{F}\left(X_{f}\right)=0$, where following the notation of [21] we define $X_{f}$ to be the value of $X$ for which $\mathcal{F}\left(X_{f}\right)=0$, and $C=X_{f} \mathcal{G}^{2}\left(X_{f}\right)$. In this case, the metric components and the scalar field read

$$
\begin{aligned}
A(r) & =-\frac{\mu}{r}+\frac{q^{2}}{2 X_{f}}\left(1+\frac{\eta}{3 \beta} r^{2}\right), \\
B(r) & =\frac{2 X_{f}}{q^{2}} A(r), \\
\psi^{\prime}(r)^{2} & =\frac{q^{2}-2 X_{f} A(r)}{A(r) B(r)},
\end{aligned}
$$

where $\mu$ is an integration constant.

With a rescaling of the time variable $q^{2}=2 X_{f}$, Eqs. (38)-(40) represent a BH solution with an effective cosmological constant $\Lambda=-\eta / \beta$ and a nontrivial profile for the scalar field. Replacing this solution into Eq. (37) we find that $\omega(r)$ satisfies the same equation (25) as in GR. The standard solution of this equation is

$$
\omega=c_{1}+\frac{c_{2}}{r^{3}},
$$

where $c_{1}$ and $c_{2}$ are integration constants which can be fixed by imposing appropriate boundary conditions.

Case 2: $\mathcal{G}=0$. Another class of solutions of Eq. (34) corresponds to choosing $\mathcal{G}\left(X_{\mathcal{G}}\right)=0$. In this case, from Eq. (37) we see that the coefficients of both $\omega^{\prime \prime}$ and $\omega^{\prime}$ vanish, and there are no corrections at linear order.

Case 3: $q=0$. Finally, we consider the case in which the scalar field is time independent $(q=0)$. Integration of the equations of motion for $A(r)$ and $B(r)$ leads to [27]

$$
\begin{aligned}
A(r)= & \frac{1}{12 \beta \zeta^{2} \eta^{2} r}\left\{r ( \zeta \eta - \beta \Lambda _ { 0 } ) \left[\zeta \eta\left(9 \beta+\eta r^{2}\right)\right.\right. \\
& \left.\left.+\beta \Lambda_{0}\left(3 \beta-\eta r^{2}\right)\right]-24 \beta \zeta^{2} \eta^{2} \mu\right\} \\
& +\frac{\sqrt{\beta}\left(\beta \Lambda_{0}+\zeta \eta\right)^{2} \arctan \left(\frac{\sqrt{\eta} r}{\sqrt{\beta}}\right)}{4 \zeta^{2} \eta^{5 / 2} r}, \\
B(r)= & \frac{4 \zeta^{2}\left(\beta+\eta r^{2}\right)^{2}}{\left(2 \beta \zeta-\beta \Lambda_{0} r^{2}+\zeta \eta r^{2}\right)^{2}} A(r),
\end{aligned}
$$

where again $\mu$ is an integration constant, while for the scalar field we obtain

$$
\psi^{\prime}(r)^{2}=-\frac{\left(\beta \Lambda_{0}+\zeta \eta\right)\left[r^{3}\left(\zeta \eta-\beta \Lambda_{0}\right)+2 \beta \zeta r\right]^{2}}{4 \beta \zeta^{2}\left(\beta+\eta r^{2}\right)^{3} A(r)} .
$$


Replacing the former expressions into Eq. (37), we find that the frame-dragging function $\omega(r)$ satisfies once again the same equation (25) as in GR.

\section{WHY THE BALDNESS?}

The no-hair theorems for static, spherically symmetric BHs proved in Refs. [29-31] rely crucially on shift symmetry, which allows us to write the equation of motion for $\phi$ as the conservation equation (28). In this section we discuss how these theorems can be generalized to the case where we consider first-order rotational corrections and timedependent scalar fields of the form (18). In this case, we can show that the nontrivial components of $J^{\mu}$ are given by

$$
\begin{aligned}
J^{r}= & B \psi^{\prime}\left[-G_{2 \mathrm{X}}+\frac{B \psi^{\prime}}{2}\left(\frac{A^{\prime}}{A}+\frac{4}{r}\right) G_{3 \mathrm{X}}\right. \\
& +\frac{2 B}{r}\left(\frac{A^{\prime}}{A}+\frac{B-1}{B r}\right) G_{4 \mathrm{X}}-\frac{2 B^{2} \psi^{\prime 2}}{r}\left(\frac{A^{\prime}}{A}+\frac{1}{r}\right) G_{4 \mathrm{XX}} \\
& \left.-\frac{B \psi^{\prime}}{2 r^{2}} \frac{A^{\prime}}{A}(3 B-1) G_{5 \mathrm{X}}+\frac{A^{\prime}}{A} \frac{B^{3} \psi^{\prime 3}}{2 r^{2}} G_{5 \mathrm{XX}}\right] \\
+ & \frac{q^{2}}{A} B \psi^{\prime}\left[\frac{2 B}{r} \frac{A^{\prime}}{A} G_{4 \mathrm{XX}}-\frac{B^{2} \psi^{\prime}}{2 r^{2}} \frac{A^{\prime}}{A} G_{5 \mathrm{XX}}\right] \\
+ & \frac{q^{2}}{A}\left[-\frac{B}{2} \frac{A^{\prime}}{A} G_{3 \mathrm{X}}+\frac{B}{2 r^{2}} \frac{A^{\prime}}{A}(B-1) G_{5 \mathrm{X}}\right] \\
\frac{A}{q} J^{t}= & G_{2 X}-\left[B \psi^{\prime \prime}+\frac{B}{2}\left(\frac{B^{\prime}}{B}+\frac{A^{\prime}}{A}+\frac{4}{r}\right) \psi^{\prime}\right] G_{3 X} \\
& -\frac{2}{r}\left(B^{\prime}+\frac{B-1}{r}\right) G_{4 X} \\
& +\frac{2 B^{2} \psi^{\prime}}{r}\left[2 \psi^{\prime \prime}+\left(\frac{B^{\prime}}{B}+\frac{A^{\prime}}{A}+\frac{1}{r}\right) \psi^{\prime}\right] G_{4 X X} \\
& +\frac{B}{r^{2}}\left[(B-1) \psi^{\prime \prime}+\frac{1}{2}\left(\frac{A^{\prime}}{A} B-\frac{B^{\prime}}{B}-\frac{A^{\prime}}{A}+3 B^{\prime}\right) \psi^{\prime}\right] G_{5 X} \\
& -\frac{B^{3} \psi^{\prime 2}}{2 r^{2}}\left[2 \psi^{\prime \prime}+\left(\frac{A^{\prime}}{A}+\frac{B^{\prime}}{B}\right) \psi^{\prime}\right] G_{5 X X} \\
= & -\frac{J^{r}}{B \psi^{\prime}}-\frac{2 A}{r}\left[\left(G_{4 X}-\frac{B \psi^{\prime}}{2 r} G_{5 X}\right)\left(\frac{B}{A}\right)^{\prime}\right. \\
& +\left(2 G_{4 X}{ }^{\prime}+\frac{B \psi^{\prime}}{2 r} G_{5 X}{ }^{\prime}-\frac{G_{5}{ }^{\prime}}{2 r \psi^{\prime}}\right) \frac{B}{A} \\
& \left.\frac{1}{2 A \psi^{\prime}}\left(r G_{3}{ }^{\prime}+\frac{G_{5}{ }^{\prime}}{r}\right)\right]
\end{aligned}
$$

For shift-symmetric theories, $G_{i}=G_{i}(X)$. These expressions can be used to extend the no-hair theorems of Refs. [29-31] to the cases considered in this paper.

For clarity and completeness, let us begin with a short summary of the original proof given in [29] (with the amendments of Refs. [30,31]).

\section{A. A review of the no-hair theorem for nonrotating black holes with a time-independent scalar field}

The no-hair theorem of Ref. [29] applies to static, spherically symmetric, asymptotically flat solutions in shift-symmetric theories. It consists of the following line of reasoning:

(1) Assuming that the scalar field $\psi(r)$ has the same symmetries as the metric (the time-dependent scalar field of [38] obviously violates this first assumption), the only nonvanishing component of $J^{\mu}$ for a spherically symmetric background is $J^{r}$, i.e. $J^{\mu}=\left(J^{r}, 0,0,0\right)$.

(2) Given a spherically symmetric space-time, defined by the line element (17) with $\omega(r)=0$, we require $J^{2}=J^{\mu} J_{\mu}$ to remain finite at the horizon $r_{h}$. Since

$$
J^{2}=\frac{\left(J^{r}\right)^{2}}{B}
$$

and $B \rightarrow 0$ for $r \rightarrow r_{h}$, this regularity condition implies that $J^{r}=0$ at the horizon.

(3) For a spherically symmetric space-time, the conservation equation (28) reduces to

$$
\frac{1}{\sqrt{-g}} \partial_{\mu}\left(\sqrt{-g} J^{\mu}\right)=\partial_{r} J^{r}+\frac{2}{r} J^{r}=0,
$$

which can be easily integrated. The solution is $J^{r} r^{2}=K$, where $K$ is an integration constant. At the horizon the areal radius $r$ cannot be zero. This implies that $K=0$, and therefore that

$$
J^{r}=0 \quad \forall r .
$$

(4) The current $J^{r}$ can be schematically written as

$$
J^{r}=B \psi^{\prime} F\left(g, g^{\prime}, g^{\prime \prime}, \psi^{\prime}\right)
$$

where $F$ is a generic function of the metric, its first and second derivatives, and $\psi^{\prime}$. At spatial infinity, asymptotic flatness implies that $B \rightarrow 1$ and $\psi^{\prime} \rightarrow 0$, while $F$ tends to a nonzero constant. This last condition is dictated by the requirement that the scalar field's kinetic energy should have the standard form: in the weak-field limit, the action contains a term that is quadratic in the field derivatives and $J_{\mu} \rightarrow \partial_{\mu} \phi$, up to an overall constant of normalization. If we now move "inward" towards the horizon, by continuity $F$ and $B$ will still be nonzero, and therefore $J^{r} \neq 0$, which contradicts Eq. (49). This contradiction can be avoided if $\psi^{\prime}=0$ for any choice of $r$, which fixes $\psi=$ constant or (without loss of generality, since the theory is shift-symmetric) $\psi=0$. 
Sotiriou and Zhou [30,31] pointed out a loophole in the last step of this proof. For Horndeski gravity theories with shift symmetry, the conserved current can be written as

$$
\begin{aligned}
J^{r}= & -B G_{2 \mathrm{X}} \psi^{\prime}+\frac{B^{2} \psi^{\prime 2}}{2}\left(\frac{A^{\prime}}{A}+\frac{4}{r}\right) G_{3 \mathrm{X}} \\
& +\frac{2 B^{2} \psi^{\prime}}{r}\left(\frac{A^{\prime}}{A}-\frac{1}{B r}+\frac{1}{r}\right) G_{4 \mathrm{X}} \\
& -\frac{2 B^{3} \psi^{\prime 3}}{r}\left(\frac{A^{\prime}}{A}+\frac{1}{r}\right) G_{4 \mathrm{XX}} \\
& -\frac{B^{3} \psi^{\prime 2}}{2 r^{2}} \frac{A^{\prime}}{A}\left(\frac{3 B-1}{B}\right) G_{5 \mathrm{X}}+\frac{A^{\prime}}{A} \frac{B^{4} \psi^{\prime 4}}{2 r^{2}} G_{5 \mathrm{XX}} .
\end{aligned}
$$

Depending on the particular form of the coupling functions $G_{i}$ we have essentially two options ${ }^{2}$ :

(a) $J^{r}$ depends linearly on $\psi^{\prime}$. This is the case considered in Ref. [29], for which $F \rightarrow-G_{2 X}$ as $r \rightarrow \infty$.

(b) $J^{r}$ contains terms which are independent of $\psi^{\prime}$, but no negative powers of $\psi^{\prime}$.

This second case represents a loophole for the no-hair theorem of Ref. [29]. Indeed, in this case the asymptotic behavior of $F$ is not trivially determined.

This is illustrated most clearly by looking at two specific examples: EdGB gravity and theories with nonminimal derivative coupling to the Einstein tensor.

In the first case the conserved current reduces to

$$
J_{\mathrm{EdGB}}^{r}=-B \psi^{\prime}-4 \alpha \frac{A^{\prime} B(B-1)}{A r^{2}},
$$

where we specialized to a linear coupling function $\xi(\phi)=\alpha \phi$ in Eq. (6), so that the theory becomes shiftsymmetric (recall that the Gauss-Bonnet combination is a topological invariant). The current (52) contains a term independent of $\psi^{\prime}$ as in case (b) above, corresponding to the loophole pointed out in Refs. [30,31]. The current vanishes at infinity, but for smaller radii the choice of $F$ is nontrivial and leads to scalar hair growth.

For the nonminimal derivative coupling theory we have instead

$$
J_{\mathrm{Gg}}^{r}=B \psi^{\prime}\left[-2 \eta+\frac{2 B}{r}\left(\frac{A^{\prime}}{A}-\frac{1}{B r}+\frac{1}{r}\right) \beta\right] .
$$

This expression for the current falls into case (a) above. The current depends linearly on $\psi^{\prime}, F \rightarrow-2 \eta$ for $r \rightarrow \infty$, and $F$ stays finite even at finite radii by continuity, as required by the arguments of [29], so we are forced to set $\psi^{\prime}=0$ and $\psi$ is a constant, which can be set to zero. Asymptotic flatness

\footnotetext{
${ }^{2}$ A third case where $J^{r}$ contains negative powers of $\psi^{\prime}$ can be excluded because it generally corresponds to theories that would not admit flat space with a trivial scalar configuration as a solution, leading to violations of local Lorentz symmetry [31].
}

was of course a key ingredient in these arguments. Hairy solutions in theories with nonminimal derivative coupling are not asymptotically flat (see e.g. [26-28]).

\section{B. Extension to slow-rotation and time-dependent scalar fields}

What is crucial for the present work is that the arguments above apply also to rotating $\mathrm{BH}$ solutions at linear order in rotation. This is because, as argued in Ref. [30], the scalar field $\phi$ (like all scalar quantities) is affected by rotation only at second order, and therefore the expression (45) for the current $J^{r}$ remains unchanged at linear order. Similarly, $J^{\theta}$ is still equal to zero at linear order. The component $J^{\varphi}$ acquires a nonzero value proportional to the $\mathrm{BH}$ angular momentum; however $J^{\varphi}$ is independent of $\varphi$, and therefore it does not contribute to the current conservation equation (28).

At first sight, the fact that no-hair theorems still hold true at linear order in rotation even for time-dependent scalar fields may be surprising. However this no-hair property can be proved through a simple extension of the arguments valid for static, nonrotating solutions. Let us extend the original argument to theories with time-dependent scalar fields of the form (18):

(1) When $\phi$ has the form (18) the current has a nonzero time component, i.e. $J^{\mu}=\left(J^{r}, 0,0, J^{t}\right)$, and its norm becomes

$$
J^{2}=\frac{\left(J^{r}\right)^{2}}{B}-\left(J^{t}\right)^{2} A
$$

(2) By imposing regularity at the horizon, where $A \rightarrow 0$, $B \rightarrow 0$, we conclude that $J^{r} \rightarrow 0$ as $r \rightarrow r_{h}$. This is true as long as $J^{t}$ does not diverge in the limit $r \rightarrow r_{h}$, i.e., as long as the quantity in square brackets in the last line of Eq. (46) is finite. For reflection-symmetric theories $\left(G_{3}=G_{5}=0\right)$, this latter requirement simplifies to the condition that $(B / A)^{\prime}$ should be finite [21].

(3) In principle, the current conservation equation (48) acquires an extra term because $J^{t} \neq 0$ :

$$
\partial_{r} J^{r}+\frac{2}{r} J^{r}+\partial_{t} J^{t}=0 .
$$

However Eq. (46) shows that in the present case $J^{t}$ is independent of time, so this term vanishes: $\partial_{t} J^{t}=0$. Following the reasoning below Eq. (48), we conclude that $J^{r}=0$ for all $r$ even for scalar fields with a linear time dependence. Note that for a timedependent scalar field, in general, the tr component of the gravitational equations $\mathcal{E}_{t r}=0$ may be nontrivial, indicating the existence of an energy flux in the radial direction. However Ref. [39] showed that, for the linear-in-time ansatz (18), $\mathcal{E}_{t r}$ is proportional 
to $J^{r}$ under the assumptions of diffeomorphism invariance and shift symmetry. Therefore the condition $J^{r}=0$ always ensures that $\mathcal{E}_{t r}=0$ : the linear time dependence (18) does not give rise to an energy flux in the radial direction.

(4) The current (45) has the form (50), where $F\left(g, g^{\prime}, g^{\prime \prime}, \psi^{\prime}\right)$ is an unspecified function. This allows us to borrow in its entirety the reasoning of Ref. [31]. We can exclude cases where $J^{r}$ contains negative powers of $\psi^{\prime}$. When all terms in $J^{r}$ contain positive powers of $\psi^{\prime}, \psi^{\prime}=0$ for all $r$ and the nohair theorem of [29] applies. The only exception is the case where $J^{r}$ contains one or more terms with no dependence on $\psi^{\prime}$, but no terms with negative powers of $\psi^{\prime}$; and then, following Sec. II B of [31], shift symmetry and Lovelock's theorem imply that the action must contain a term proportional to the Gauss-Bonnet invariant.

This generalized no-hair theorem can be used to justify the absence of corrections to GR at linear order that we found in Sec. III. For a theory with nonminimal derivative coupling to the Einstein tensor, the nonzero components of the current can be obtained by specializing Eqs. (45)-(46), with the result

$$
\begin{aligned}
& J_{\mathrm{Gg}}^{r}=B \psi^{\prime}\left[-2 \eta+\frac{2 B}{r}\left(\frac{A^{\prime}}{A}-\frac{1}{B r}+\frac{1}{r}\right) \beta\right], \\
& \frac{A}{q} J_{\mathrm{Gg}}^{t}=2 \eta+\frac{2 B}{r}\left(\frac{1-B}{B r}-\frac{B^{\prime}}{B}\right) \beta .
\end{aligned}
$$

The $J^{r}$ component is identical to the static case of Eq. (53), it does not contain any $\psi^{\prime}$-independent terms, and the nohair theorem of [29] implies that asymptotically flat solutions must be the same as GR.

In conclusion, the only no-hair violations at linear order in rotation when the scalar field depends linearly on time and when we require asymptotic flatness can occur in one of two cases:

(i) if the scalar field has a linear coupling to the GaussBonnet invariant, or

(ii) if, as proposed in Ref. [38], the field equations of the theory guarantee that the current vanishes identically $\left(J^{r}=0\right)$ because $F\left(g, g^{\prime}, g^{\prime \prime}, \psi^{\prime}\right)=0$ as a consequence of the field equations. Note that this is only possible for special forms of the function $G_{i}$, and that the scalar field must then be time dependent (i.e., it must violate some of the symmetries of the metric) in order to be regular at the horizon.

\section{CONCLUSIONS}

In this work we studied leading-order rotational corrections to a broad class of $\mathrm{BH}$ solutions in Horndeski gravity. With the known exception of EdGB gravity [30-35], we have found that the frame-dragging function $\omega(r)$, which describes the leading-order rotational corrections, is exactly the same as in GR for all of the Horndeski BH solutions known in the literature. This result applies even to asymptotically flat solutions that violate the no-hair theorems by requiring the scalar field to be time dependent (so that the scalar field does not respect the same symmetries as the metric), as proposed in Ref. [38].

The formalism developed in this paper can be extended in various directions. First of all, the no-hair theorem proved in Sec. IV at first order in rotation is not expected to hold at second order, where the continuity equation will be modified. Calculations of $\mathrm{BH}$ solutions at second order in rotation, along the lines of [34,35], are already underway [45].

Even for nonrotating Horndeski BHs, studies of stability and perturbative dynamics (as encoded in their quasinormal mode spectrum; see e.g. [46] for a review) are still in their infancy. One of us [47] studied massless scalar field perturbations of static $\mathrm{BH}$ solutions in theories with field derivative coupling to the Einstein tensor. More in general, gravitational perturbations of static, nonrotating spacetimes can be explored using the formalism developed in Refs. $[43,44]$. The present work lays the foundations to study quasinormal modes and look for super-radiant instabilities using the slow-rotation perturbative techniques reviewed, e.g., in Ref. [48].

Another important extension concerns compact stars in Horndeski gravity. Slowly rotating compact stars in EdGB gravity were studied in [49]. Cisterna et al. [50] investigated compact objects in theories with a nonminimal derivative coupling of the scalar field with the Einstein tensor. Our formalism can be extended relatively easily to study compact stars in broader classes of Horndeski gravity, and to understand whether genuine strong-field deviations from GR (similar to the "spontaneous scalarization" phenomena proposed by Damour and EspositoFarèse [51]) can occur in some sectors of the Horndeski gravity action; see e.g. [52] for recent work in this direction.

\section{ACKNOWLEDGMENTS}

We thank Eugeny Babichev, Vitor Cardoso, Sante Carloni, Adolfo Cisterna, Leonardo Gualtieri, Matteo Lulli, Paolo Pani and Eleftherios Papantonopoulos for discussions. A.M. was supported by NSF Grants No. 1205864, No. 1212433 and No. 1333360. E. B. was supported by NSF CAREER Grant No. PHY-1055103 and by FCT Grant No. IF/00797/2014/CP1214/CT0012 under the IF2014 program. H. O.S. was supported by NSF CAREER Grant No. PHY-1055103 and by a summer research assistantship award from the University of Mississippi. E. B. and H. O.S. thank the Instituto Superior Técnico (Lisbon, Portugal), where part of this project was completed, for the hospitality. M. M. was supported by the FCT Portugal through Grant No. SFRH/BPD/88299/2012. 


\section{APPENDIX: FIELD EQUATIONS}

In this appendix we list the left-hand side of the field equations. For clarity, we split all of the left-hand sides of the field equations as a sum of two contributions, so that the case of time-independent scalar fields can more easily be recovered by setting $q=0$ :

$$
\mathcal{E}_{\alpha \beta}=\mathcal{E}_{\alpha \beta}^{(0)}+\frac{q^{2}}{A} \mathcal{E}_{\alpha \beta}^{(t)},
$$

$$
\mathcal{E}_{\phi}=\mathcal{E}_{\phi}^{(0)}+\frac{q^{2}}{A} \mathcal{E}_{\phi}^{(t)}
$$

Let us remark that the equations of motion still depend on the specific form of the functions $G_{i}$, which are functions of the kinetic energy (19), and therefore may contain $q$-dependent terms; therefore we must evaluate all of the functions $G_{i}$ at $q=0$ to recover the time-independent limit. The explicit forms of the various terms are

$$
\begin{aligned}
& \mathcal{E}_{t t}^{(0)}=G_{2}+B \psi^{\prime 2} G_{3 \phi}-\frac{B \psi^{\prime 2}}{2}\left(B^{\prime} \psi^{\prime}+2 B \psi^{\prime \prime}\right) G_{3 \mathrm{X}}-\frac{2}{r}\left(\frac{B-1}{r}+B^{\prime}\right) G_{4}-\frac{2 B^{2} \psi^{\prime}}{r}\left(\frac{\psi^{\prime}}{r}+2 \frac{B^{\prime}}{B} \psi^{\prime}+2 \psi^{\prime \prime}\right) G_{4 \mathrm{X}} \\
& -B\left(\frac{4}{r} \psi^{\prime}+\frac{B^{\prime}}{B} \psi^{\prime}+2 \psi^{\prime \prime}\right) G_{4 \phi}+\frac{2 B^{2} \psi^{\prime 3}}{r}\left(B^{\prime} \psi^{\prime}+2 B \psi^{\prime \prime}\right) G_{4 \mathrm{XX}}-B^{2} \psi^{\prime 2}\left(\frac{4}{r} \psi^{\prime}-\frac{B^{\prime}}{B} \psi^{\prime}-2 \psi^{\prime \prime}\right) G_{4 \mathrm{X} \phi} \\
& -2 B \psi^{\prime 2} G_{4 \phi \phi}+\frac{B \psi^{\prime 2}}{2 r^{2}}\left(5 B^{\prime} B \psi^{\prime}+6 B^{2} \psi^{\prime \prime}-B^{\prime} \psi^{\prime}-2 B \psi^{\prime \prime}\right) G_{5 \mathrm{X}}+\frac{B^{3} \psi^{\prime 3}}{r}\left(\frac{\psi^{\prime}}{r}-\frac{B^{\prime}}{B} \psi^{\prime}-2 \psi^{\prime \prime}\right) G_{5 \mathrm{X} \phi} \\
& -\frac{B^{3} \psi^{\prime 4}}{2 r^{2}}\left(B^{\prime} \psi^{\prime}+2 B \psi^{\prime \prime}\right) G_{5 \mathrm{XX}}+\frac{B \psi^{\prime}}{r}\left(3 B^{\prime} \psi^{\prime}+4 B \psi^{\prime \prime}+\frac{\psi^{\prime}}{r}+B \frac{\psi^{\prime}}{r}\right) G_{5 \phi}+\frac{2 B^{2} \psi^{\prime 3}}{r} G_{5 \phi \phi}, \\
& \mathcal{E}_{t t}^{(t)}=-G_{2 \mathrm{X}}+G_{3 \phi}+\frac{B}{2}\left(4 \frac{\psi^{\prime}}{r}+\frac{B^{\prime}}{B} \psi^{\prime}+2 \psi^{\prime \prime}\right) G_{3 \mathrm{X}}+\frac{2}{r}\left(\frac{B-1}{r}+B^{\prime}\right) G_{4 \mathrm{X}}-\left(\frac{4 B}{r} \psi^{\prime}+B^{\prime} \psi^{\prime}+2 B \psi^{\prime \prime}\right) G_{4 \mathrm{X} \phi} \\
& -\frac{2 B \psi^{\prime}}{r}\left(B^{\prime} \psi^{\prime}+2 B \psi^{\prime \prime}+\frac{B}{r} \psi^{\prime}\right) G_{4 \mathrm{XX}}-\frac{1}{2 r^{2}}\left(3 B^{\prime} B \psi^{\prime}-B^{\prime} \psi^{\prime}-2 B \psi^{\prime \prime}+2 B^{2} \psi^{\prime \prime}\right) G_{5 \mathrm{X}}-\frac{1}{r}\left(\frac{B-1}{r}+B^{\prime}\right) G_{5 \phi} \\
& +\frac{B \psi^{\prime}}{r}\left(B^{\prime} \psi^{\prime}+2 B \psi^{\prime \prime}+\frac{B}{r} \psi^{\prime}\right) G_{5 \mathrm{X} \phi}+\frac{B^{2} \psi^{\prime 2}}{2 r^{2}}\left(B^{\prime} \psi^{\prime}+2 B \psi^{\prime \prime}\right) G_{5 \mathrm{XX}}, \\
& \mathcal{E}_{r r}^{(0)}=G_{2}+B \psi^{\prime 2} G_{2 X}-B \psi^{\prime 2} G_{3 \phi}-\frac{B^{2} \psi^{\prime 3}}{2}\left(\frac{4}{r}+\frac{A^{\prime}}{A}\right) G_{3 \mathrm{X}}-\frac{2}{r}\left(B \frac{A^{\prime}}{A}+\frac{B-1}{r}\right) G_{4}-B \psi^{\prime}\left(\frac{4}{r}+\frac{A^{\prime}}{A}\right) G_{4 \phi} \\
& -\frac{2 B \psi^{\prime 2}}{r}\left(2 B \frac{A^{\prime}}{A}+\frac{2 B-1}{r}\right) G_{4 \mathrm{X}}+B^{2} \psi^{\prime 3}\left(\frac{4}{r}+\frac{A^{\prime}}{A}\right) G_{4 \mathrm{X} \phi}+\frac{2 B^{3} \psi^{\prime 4}}{r}\left(\frac{A^{\prime}}{A}+\frac{1}{r}\right) G_{4 \mathrm{XX}} \\
& +\frac{B \psi^{\prime 2}}{r}\left(3 B \frac{A^{\prime}}{A}+\frac{3 B-1}{r}\right) G_{5 \phi}+\frac{B^{2} \psi^{\prime 3}}{2 r^{2}} \frac{A^{\prime}}{A}(5 B-1) G_{5 \mathrm{X}}-\frac{B^{3} \psi^{\prime 4}}{r}\left(\frac{A^{\prime}}{A}+\frac{1}{r}\right) G_{5 \mathrm{X} \phi}-\frac{B^{4} \psi^{\prime 5}}{2 r^{2}} \frac{A^{\prime}}{A} G_{5 \mathrm{XX}}, \\
& \mathcal{E}_{r r}^{(t)}=-G_{3 \phi}+\frac{B \psi^{\prime}}{2} \frac{A^{\prime}}{A} G_{3 \mathrm{X}}+\frac{2 B}{r} \frac{A^{\prime}}{A} G_{4 \mathrm{X}}+2 G_{4 \phi \phi}-\frac{2 B^{2} \psi^{\prime 2}}{r} \frac{A^{\prime}}{A} G_{4 \mathrm{XX}}+B \psi^{\prime}\left(\frac{4}{r}-\frac{A^{\prime}}{A}\right) G_{4 \mathrm{X} \phi}-\frac{2 B \psi^{\prime}}{r} G_{5 \phi \phi} \\
& -\frac{B \psi^{\prime}}{2 r^{2}} \frac{A^{\prime}}{A}(3 B-1) G_{5 \mathrm{X}}+\frac{B^{2} \psi^{\prime 2}}{r}\left(\frac{A^{\prime}}{A}-\frac{1}{r}\right) G_{5 \mathrm{X} \phi}+\frac{B^{3} \psi^{\prime 3}}{2 r^{2}} G_{5 \mathrm{XX}}+\frac{1}{r}\left(\frac{B-1}{r}-B \frac{A^{\prime}}{A}\right) G_{5 \phi},
\end{aligned}
$$




$$
\begin{aligned}
& \mathcal{E}_{t \varphi}^{(0)}=\omega G_{2}+B \psi^{\prime 2} \omega G_{3 \phi}-\frac{B \psi^{\prime 2}}{2} \omega\left(B^{\prime} \psi^{\prime}+2 B \psi^{\prime \prime}\right) G_{3 \mathrm{X}}+\frac{B}{2}\left[-\left(\frac{2}{r} \frac{B^{\prime}}{B}+2 \frac{A^{\prime \prime}}{A}+\frac{B^{\prime}}{B} \frac{A^{\prime}}{A}-\frac{A^{\prime 2}}{A^{2}}+\frac{2 A^{\prime}}{r} \frac{1}{A}\right) \omega\right. \\
& \left.+\left(\frac{B^{\prime}}{B}+\frac{8}{r}-\frac{A^{\prime}}{A}\right) \omega^{\prime}+2 \omega^{\prime \prime}\right] G_{4}-\left[\left(\frac{A^{\prime}}{A} B \psi^{\prime}+B^{\prime} \psi^{\prime}+2 B \psi^{\prime \prime}+\frac{2}{r} B \psi^{\prime}\right) \omega-\omega^{\prime} B \psi^{\prime}\right] G_{4 \phi} \\
& +\frac{B^{2} \psi^{\prime}}{2}\left[-\left(\frac{2}{r} \frac{A^{\prime}}{A} \psi^{\prime}+\frac{4}{r} \frac{B^{\prime}}{B} \psi^{\prime}+2 \frac{A^{\prime \prime}}{A} \psi^{\prime}-\frac{A^{\prime 2}}{A^{2}} \psi^{\prime}+2 \frac{B^{\prime}}{B} \frac{A^{\prime}}{A} \psi^{\prime}+2 \frac{A^{\prime}}{A} \psi^{\prime \prime}+\frac{4}{r} \psi^{\prime \prime}\right) \omega+2 \psi^{\prime} \omega^{\prime \prime}\right. \\
& \left.+\left(\frac{8 \psi^{\prime}}{r}+\frac{2 B^{\prime}}{B} \psi^{\prime}+2 \psi^{\prime \prime}-\frac{A^{\prime}}{A} \psi^{\prime}\right) \omega^{\prime}\right] G_{4 \mathrm{X}}+B^{2} \psi^{\prime 2}\left[\left(\frac{B^{\prime}}{B} \psi^{\prime}-\frac{A^{\prime}}{A} \psi^{\prime}+2 \psi^{\prime \prime}-\frac{2}{r} \psi^{\prime}\right) \omega+\psi^{\prime} \omega^{\prime \prime}\right] G_{4 \mathrm{X} \phi} \\
& -2 B \psi^{\prime 2} \omega G_{4 \phi \phi}+\frac{B^{2} \psi^{\prime 3}}{2}\left[\left(\frac{2}{r} \psi^{\prime} B^{\prime}+\psi^{\prime} B^{\prime} \frac{A^{\prime}}{A}+\frac{4}{r} B \psi^{\prime} \psi^{\prime \prime}+2 B \frac{A^{\prime}}{A} \psi^{\prime \prime}\right) \omega-\left(\psi^{\prime} B^{\prime}+2 B \psi^{\prime \prime}\right) \omega^{\prime}\right] G_{4 \mathrm{XX}} \\
& +\frac{B^{3} \psi^{\prime 2}}{4 r}\left[-\left(5 \frac{B^{\prime}}{B} \psi^{\prime}-\frac{A^{\prime}}{A} \psi^{\prime}+6 \psi^{\prime \prime}+\frac{6}{r} \psi^{\prime}\right) \omega^{\prime}+\left(5 \frac{B^{\prime}}{B} \frac{A^{\prime}}{A} \psi^{\prime}-\frac{A^{\prime 2}}{A^{2}} \psi^{\prime}+6 \frac{A^{\prime}}{A} \psi^{\prime \prime}+2 \frac{A^{\prime \prime}}{A} \psi^{\prime}\right) \omega-2 \psi^{\prime} \omega^{\prime \prime}\right] G_{5 \mathrm{X}} \\
& +\frac{B^{2} \psi^{\prime 3}}{4}\left[\left(\psi^{\prime} B^{\prime}+2 B \psi^{\prime \prime}-\frac{2 B}{r} \psi^{\prime}\right) \omega^{\prime}-\left(\psi^{\prime} B^{\prime} \frac{A^{\prime}}{A}+2 B \frac{A^{\prime}}{A} \psi^{\prime \prime}-\frac{2}{r} B \psi^{\prime} \frac{A^{\prime}}{A}+\frac{2}{r} \psi^{\prime} B^{\prime}+\frac{4}{r} B \psi^{\prime \prime}\right) \omega\right] G_{5 \mathrm{X} \phi} \\
& +\frac{B^{3} \psi^{\prime 4}}{4 r}\left[\left(\psi^{\prime} B^{\prime}+2 B \psi^{\prime \prime}\right) \omega^{\prime}-\frac{A^{\prime}}{A}\left(\psi^{\prime} B^{\prime}+2 B \psi^{\prime \prime}\right) \omega\right] G_{5 \mathrm{XX}}+\frac{B \psi^{\prime}}{4}\left[\left(\frac{A^{\prime}}{A} B \psi^{\prime}-\frac{8}{r} B \psi^{\prime}-4 B \psi^{\prime \prime}-3 B^{\prime} \psi^{\prime}\right) \omega^{\prime}\right. \\
& \left.+\left(3 B^{\prime} \frac{A^{\prime}}{A} \psi^{\prime}-\frac{B A^{\prime 2}}{A^{2}} \psi^{\prime}+\frac{2}{r} \frac{A^{\prime}}{A} B \psi^{\prime}+2 B \frac{A^{\prime \prime}}{A} \psi^{\prime}+\frac{6}{r} B^{\prime} \psi^{\prime}+\frac{8}{r} B \psi^{\prime \prime}+4 \frac{A^{\prime}}{A} B \psi^{\prime \prime}\right) \omega-2 B \psi^{\prime} \omega^{\prime \prime}\right] G_{5 \phi} \\
& +\frac{B^{2} \psi^{\prime 3}}{2}\left[\left(\frac{2}{r}+\frac{A^{\prime}}{A}\right) \omega-\omega^{\prime}\right] G_{5 \phi \phi},
\end{aligned}
$$

$$
\begin{aligned}
& \mathcal{E}_{t \varphi}^{(t)}=-\frac{B \psi^{\prime}}{2} \frac{A^{\prime}}{A} \omega G_{3 \mathrm{X}}-\omega G_{3 \phi}+\left[\left(\frac{2 B}{r} \frac{A^{\prime}}{A}+2 B \frac{A^{\prime \prime}}{A}-2 B \frac{A^{\prime 2}}{A^{2}}+B^{\prime} \frac{A^{\prime}}{A}\right) \frac{\omega}{2}-\left(B^{\prime}+\frac{8 B}{r}-2 B \frac{A^{\prime}}{A}\right) \frac{\omega^{\prime}}{2}-B \omega^{\prime \prime}\right] G_{4 \mathrm{X}} \\
& +\left[\left(3 \frac{A^{\prime}}{A} B \psi^{\prime}+\frac{2 B}{r} \psi^{\prime}+B^{\prime} \psi^{\prime}+2 B \psi^{\prime \prime}\right) \omega-\omega^{\prime} B \psi^{\prime}\right] G_{4 X \phi}+\frac{B}{2}\left[B \psi^{\prime}\left(\frac{B^{\prime}}{B} \psi^{\prime}+2 \psi^{\prime \prime}-\psi^{\prime} \frac{A^{\prime}}{A}\right) \omega^{\prime}\right. \\
& \left.-\frac{A^{\prime}}{A} B \psi^{\prime}\left(\frac{B^{\prime}}{B} \psi^{\prime}-\frac{A^{\prime}}{A} \psi^{\prime}+2 \psi^{\prime \prime}-\frac{2}{r} \psi^{\prime}\right) \omega\right] G_{4 \mathrm{XX}}+2 \omega G_{4 \phi \phi}+\left[\frac{B^{2}}{4 r}\left(\frac{6 \psi^{\prime}}{r}-3 \frac{A^{\prime}}{A} \psi^{\prime}+3 \frac{B^{\prime}}{B} \psi^{\prime}+2 \psi^{\prime \prime}\right) \omega^{\prime}\right. \\
& \left.-\frac{B^{2}}{4 r}\left(3 \frac{B^{\prime}}{B} \frac{A^{\prime}}{A} \psi^{\prime}+2 \frac{A^{\prime}}{A} \psi^{\prime \prime}+2 \frac{A^{\prime \prime}}{A} \psi^{\prime}-3 \frac{A^{\prime 2}}{A^{2}} \psi^{\prime}\right) \omega+\frac{B^{2} \psi^{\prime}}{2 r} \omega^{\prime \prime}\right] G_{5 \mathrm{X}}+\left[\frac{B^{2} \psi^{\prime}}{4}\left(\frac{A^{\prime}}{A} \psi^{\prime}-\frac{B^{\prime}}{B} \psi^{\prime}-2 \psi^{\prime \prime}+\frac{2}{r} \psi^{\prime}\right) \omega^{\prime}\right. \\
& \left.-\frac{B^{2} \psi^{\prime}}{4}\left(\frac{A^{\prime 2}}{A^{2}} \psi^{\prime}-\frac{B^{\prime}}{B} \frac{A^{\prime}}{A} \psi^{\prime}-2 \frac{A^{\prime}}{A} \psi^{\prime \prime}+\frac{6}{r} \frac{A^{\prime}}{A} \psi^{\prime}+\frac{2}{r} \frac{B^{\prime}}{B} \psi^{\prime}+\frac{4}{r} \psi^{\prime \prime}\right) \omega\right] G_{5 \mathrm{X} \phi}+\frac{B^{3} \psi^{\prime 2}}{4 r} \frac{A^{\prime}}{A}\left[\left(\psi^{\prime}-\frac{A B^{\prime}}{A^{\prime} B} \psi^{\prime}-\frac{2 A}{A^{\prime}} \psi^{\prime \prime}\right) \omega^{\prime}\right. \\
& \left.+\left(\frac{B^{\prime} \psi^{\prime}}{B}-\frac{A^{\prime} \psi^{\prime}}{A}+2 \psi^{\prime \prime}\right) \omega\right] G_{5 \mathrm{XX}}+\frac{B}{4}\left[\left(-\frac{2 A^{\prime}}{r} \frac{A^{\prime 2}}{A}+3 \frac{A^{\prime \prime}}{A^{2}}-2 \frac{2}{A}+\frac{B^{\prime}}{B}-\frac{B^{\prime}}{B} \frac{A^{\prime}}{A}\right) \omega\right. \\
& \left.+\left(\frac{8}{r}+\frac{B^{\prime}}{B}-3 \frac{A^{\prime}}{A}\right) \omega^{\prime}+2 \omega^{\prime \prime}\right] G_{5 \phi}+\frac{B}{2}\left[-\left(2 \frac{A^{\prime}}{A} \psi^{\prime}+\frac{B^{\prime}}{B} \psi^{\prime}+2 \psi^{\prime \prime}+\frac{2}{r} \psi^{\prime}\right) \omega+\psi^{\prime} \omega^{\prime}\right] G_{5 \phi \phi} \\
& +\frac{q^{2}}{A} \frac{B}{4} \frac{A^{\prime}}{A}\left(\frac{A^{\prime}}{A} \omega-\omega^{\prime}\right)\left(G_{5 \mathrm{X} \phi}-2 G_{4 \mathrm{XX}}+\frac{B \psi^{\prime}}{r} G_{5 \mathrm{XX}}\right) \text {, }
\end{aligned}
$$




$$
\begin{aligned}
& \mathcal{E}_{\phi}^{(0)}=G_{2 \phi}+B \psi^{\prime}\left(\frac{A^{\prime}}{2 A}+\frac{B^{\prime}}{2 B}+\frac{2}{r}+\frac{\psi^{\prime \prime}}{\psi^{\prime}}\right) G_{2 \mathrm{X}}+B \psi^{\prime 2} G_{2 \mathrm{X} \phi}-B \psi^{\prime 3}\left(\frac{B^{\prime}}{2}+\frac{B \psi^{\prime \prime}}{\psi^{\prime}}\right) G_{2 \mathrm{XX}} \\
& -B \psi^{\prime}\left(\frac{B^{\prime}}{B}+\frac{A^{\prime}}{A}+\frac{4}{r}+\frac{2 \psi^{\prime \prime}}{\psi^{\prime}}\right) G_{3 \phi}-B^{2} \psi^{\prime 2}\left(\frac{3 A^{\prime} B^{\prime}}{4 A B}+\frac{3 B^{\prime}}{B r}+\frac{A^{\prime} \psi^{\prime \prime}}{A \psi^{\prime}}+\frac{4 \psi^{\prime \prime}}{\psi^{\prime} r}+\frac{A^{\prime \prime}}{2 A}-\frac{A^{\prime 2}}{4 A^{2}}+\frac{2 A^{\prime}}{A r}+\frac{2}{r^{2}}\right) G_{3 \mathrm{X}} \\
& +B^{2} \psi^{\prime 3}\left(\frac{\psi^{\prime \prime}}{\psi^{\prime}}-\frac{A^{\prime}}{2 A}+\frac{B^{\prime}}{2 B}-\frac{2}{r}\right) G_{3 \mathrm{X} \phi}+B^{3} \psi^{\prime 4}\left(\frac{B^{\prime}}{B r}+\frac{A^{\prime} B^{\prime}}{4 A B}+\frac{2 \psi^{\prime \prime}}{r \psi^{\prime}}+\frac{A^{\prime} \psi^{\prime \prime}}{2 A \psi^{\prime}}\right) G_{3 \mathrm{XX}}-B \psi^{\prime 2} G_{3 \phi \phi} \\
& +B\left(\frac{A^{\prime 2}}{2 A^{2}}-\frac{A^{\prime \prime}}{A}-\frac{A^{\prime} B^{\prime}}{2 A B}-\frac{2 A^{\prime}}{A r}-\frac{2 B^{\prime}}{B r}-\frac{2}{r^{2}}+\frac{2}{B r^{2}}\right) G_{4 \phi} \\
& +B^{2} \psi^{\prime}\left(\frac{A^{\prime 2}}{A^{2} r}-\frac{2 A^{\prime \prime}}{A r}-\frac{3 A^{\prime} B^{\prime}}{A B r}-\frac{3 A^{\prime}}{A r^{2}}-\frac{3 B^{\prime}}{B r^{2}}+\frac{A^{\prime}}{A B r^{2}}-\frac{2 A^{\prime} \psi^{\prime \prime}}{A \psi^{\prime} r}-\frac{2 \psi^{\prime \prime}}{\psi^{\prime} r^{2}}+\frac{B^{\prime}}{B^{2} r^{2}}+\frac{2 \psi^{\prime \prime}}{B \psi^{\prime} r^{2}}\right) G_{4 \mathrm{X}} \\
& +B^{3} \psi^{\prime 3}\left(\frac{2 A^{\prime \prime}}{A r}-\frac{A^{\prime 2}}{A^{2} r}+\frac{6 A^{\prime} B^{\prime}}{A B r}+\frac{3 A^{\prime}}{A r^{2}}+\frac{6 B^{\prime}}{B r^{2}}+\frac{8 A^{\prime} \psi^{\prime \prime}}{A \psi^{\prime} r}+\frac{8 \psi^{\prime \prime}}{\psi^{\prime} r^{2}}-\frac{B^{\prime}}{B^{2} r^{2}}-\frac{2 \psi^{\prime \prime}}{B \psi^{\prime} r^{2}}\right) G_{4 \mathrm{XX}} \\
& +B^{2} \psi^{\prime 2}\left(\frac{A^{\prime \prime}}{A}-\frac{A^{\prime 2}}{2 A^{2}}+\frac{2 A^{\prime} B^{\prime}}{A B}+\frac{4 A^{\prime}}{A r}+\frac{8 B^{\prime}}{B r}+\frac{4}{r^{2}}+\frac{2}{B r^{2}}+\frac{3 A^{\prime} \psi^{\prime \prime}}{A \psi^{\prime}}+\frac{12 \psi^{\prime \prime}}{\psi^{\prime} r}\right) G_{4 \mathrm{X} \phi}+B^{2} \psi^{\prime 3}\left(\frac{A^{\prime}}{A}+\frac{4}{r}\right) G_{4 \mathrm{X} \phi \phi} \\
& +B^{3} \psi^{\prime 4}\left(\frac{2 A^{\prime}}{A r}-\frac{2 B^{\prime}}{B r}-\frac{A^{\prime} B^{\prime}}{2 A B}-\frac{A^{\prime} \psi^{\prime \prime}}{A \psi^{\prime}}-\frac{4 \psi^{\prime \prime}}{\psi^{\prime} r}+\frac{2}{r^{2}}\right) G_{4 \mathrm{XX} \phi}-B^{3} \psi^{\prime 5}\left(\frac{A^{\prime} B^{\prime}}{A r}+\frac{B^{\prime}}{r^{2}}+\frac{2 A^{\prime} B \psi^{\prime \prime}}{A \psi^{\prime} r}+\frac{2 B \psi^{\prime \prime}}{\psi^{\prime} r^{2}}\right) G_{4 \mathrm{XXX}} \\
& +B^{2} \psi^{\prime}\left(\frac{2 A^{\prime \prime}}{A r}-\frac{A^{\prime 2}}{A^{2} r}+\frac{3 A^{\prime}}{A r^{2}}+\frac{3 A^{\prime} B^{\prime}}{A B r}+\frac{3 B^{\prime}}{B r^{2}}-\frac{A^{\prime}}{A B r^{2}}+\frac{2 A^{\prime} \psi^{\prime \prime}}{A \psi^{\prime} r}+\frac{2 \psi^{\prime \prime}}{\psi^{\prime} r^{2}}-\frac{B^{\prime}}{B^{2} r^{2}}-\frac{2 \psi^{\prime \prime}}{\psi^{\prime} B r^{2}}\right) G_{5 \phi} \\
& +B^{3} \psi^{\prime 2}\left(\frac{3 A^{\prime \prime}}{2 A r^{2}}-\frac{3 A^{\prime 2}}{4 A^{2} r^{2}}+\frac{15 A^{\prime} B^{\prime}}{4 A B r^{2}}+\frac{A^{\prime 2}}{4 A^{2} B r^{2}}-\frac{A^{\prime \prime}}{2 A B r^{2}}-\frac{3 A^{\prime} B^{\prime}}{4 A B^{2} r^{2}}+\frac{3 A^{\prime} \psi^{\prime \prime}}{A \psi^{\prime} r^{2}}-\frac{A^{\prime} \psi^{\prime \prime}}{A B \psi^{\prime} r^{2}}\right) G_{5 \mathrm{X}} \\
& +B^{4} \psi^{\prime 4}\left(\frac{A^{\prime 2}}{4 A^{2} r^{2}}-\frac{A^{\prime \prime}}{2 A r^{2}}-\frac{5 A^{\prime} B^{\prime}}{2 A B r^{2}}-\frac{7 A^{\prime} \psi^{\prime \prime}}{2 \psi^{\prime} A r^{2}}+\frac{A^{\prime} B^{\prime}}{4 A B^{2} r^{2}}+\frac{A^{\prime} \psi^{\prime \prime}}{2 A B \psi^{\prime} r^{2}}\right) G_{5 \mathrm{XX}} \\
& +B^{3} \psi^{\prime 3}\left(\frac{A^{\prime 2}}{2 A^{2} r}-\frac{A^{\prime \prime}}{A r}-\frac{7 A^{\prime} B^{\prime}}{2 A B r}-\frac{A^{\prime}}{2 A r^{2}}-\frac{7 B^{\prime}}{2 B r^{2}}-\frac{A^{\prime}}{2 A B r^{2}}-\frac{5 A^{\prime} \psi^{\prime \prime}}{A \psi^{\prime} r}-\frac{5 \psi^{\prime \prime}}{\psi^{\prime} r^{2}}+\frac{B^{\prime}}{2 B^{2} r^{2}}+\frac{\psi^{\prime \prime}}{\psi^{\prime} B r^{2}}\right) G_{5 \mathrm{X} \phi} \\
& +B^{2} \psi^{\prime 2}\left(\frac{A^{\prime}}{A r}-\frac{1}{B r^{2}}+\frac{1}{r^{2}}\right) G_{5 \phi \phi}-B^{3} \phi^{\prime 4}\left(\frac{A^{\prime}}{A r}+\frac{1}{r^{2}}\right) G_{5 \mathrm{X} \phi \phi} \\
& +B^{4} \phi^{\prime 5}\left(\frac{A^{\prime} B^{\prime} \psi^{\prime}}{2 A B r}-\frac{A^{\prime}}{2 A r^{2}}+\frac{B^{\prime}}{2 B r^{2}}+\frac{A^{\prime} \psi^{\prime \prime}}{A \psi^{\prime} r}+\frac{\psi^{\prime \prime}}{\psi^{\prime} r^{2}}\right) G_{5 \mathrm{XX} \phi}+B^{4} \psi^{6 \prime}\left(\frac{A^{\prime} B^{\prime}}{4 A r^{2}}+\frac{A^{\prime} B \psi^{\prime \prime}}{2 A r^{2} \psi^{\prime}}\right) G_{5 \mathrm{XXX}},
\end{aligned}
$$

$$
\begin{aligned}
\mathcal{E}_{\phi}^{(t)}= & -G_{2 \mathrm{X} \phi}-\frac{A^{\prime} B \psi^{\prime}}{2 A} G_{2 \mathrm{XX}}+\left(\frac{B A^{\prime}}{A r}-\frac{3 B A^{\prime 2}}{4 A^{2}}+\frac{A^{\prime} B^{\prime}}{4 A}+\frac{B A^{\prime \prime}}{2 A}\right) G_{3 \mathrm{X}}+G_{3 \phi \phi}+B \psi^{\prime}\left(\frac{B^{\prime}}{2 B}+\frac{2}{r}+\frac{3 A^{\prime}}{2 A}+\frac{\psi^{\prime \prime}}{\psi^{\prime}}\right) G_{3 \mathrm{X} \phi} \\
& +B^{2} \psi^{\prime 2}\left(\frac{A^{\prime}}{A r}+\frac{A^{\prime 2}}{4 A^{2}}-\frac{A^{\prime} \psi^{\prime \prime}}{2 A \psi^{\prime}}-\frac{A^{\prime} B^{\prime}}{4 A B}\right) G_{3 \mathrm{XX}}+B^{2} \psi^{\prime}\left(\frac{4 A^{\prime 2}}{A^{2} r}-\frac{2 A^{\prime \prime}}{A r}-\frac{3 A^{\prime} B^{\prime}}{A B r}-\frac{A^{\prime}}{A r}-\frac{2 A^{\prime} \psi^{\prime \prime}}{A \psi^{\prime} r}-\frac{A^{\prime}}{A B r^{2}}\right) G_{4 \mathrm{XX}} \\
& +\left(\frac{2 A^{\prime 2} B}{A^{2}}-\frac{B A^{\prime \prime}}{A}-\frac{A^{\prime} B^{\prime}}{2 A}-\frac{2 A^{\prime} B}{A r}+\frac{2 B^{\prime}}{r}+\frac{2(B-1)}{r^{2}}\right) G_{4 \mathrm{X} \phi}-B \psi^{\prime}\left(\frac{2 A^{\prime}}{A}+\frac{B^{\prime}}{B}+\frac{2 \psi^{\prime \prime}}{\psi^{\prime}}+\frac{4}{r}\right) G_{4 \mathrm{X} \phi \phi} \\
& -B^{2} \psi^{\prime 2}\left(\frac{6 A^{\prime}}{A r}+\frac{2 B^{\prime}}{B r}-\frac{A^{\prime} B^{\prime}}{2 A B}-\frac{A^{\prime} \psi^{\prime \prime}}{A \psi^{\prime}}+\frac{A^{\prime 2}}{2 A^{2}}+\frac{2}{r^{2}}+\frac{4 \psi^{\prime \prime}}{\psi^{\prime} r}\right) G_{4 \mathrm{XX} \phi}+B^{2} \psi^{\prime 3}\left(\frac{A^{\prime} B^{\prime}}{A r}+\frac{2 A^{\prime} B \psi^{\prime \prime}}{A \psi^{\prime} r}-\frac{A^{\prime 2} B}{A^{2} r}-\frac{A^{\prime} B}{A r^{2}}\right) G_{4 \mathrm{XXX}} \\
& +B^{2}\left(\frac{3 A^{\prime 2}}{4 A^{2} r^{2}}-\frac{A^{\prime \prime}}{2 A r^{2}}-\frac{3 A^{\prime} B^{\prime}}{4 A B r^{2}}-\frac{3 A^{\prime 2}}{4 A^{2} B r^{2}}+\frac{A^{\prime \prime}}{2 A B r^{2}}+\frac{A^{\prime} B^{\prime}}{4 A B^{2} r^{2}}\right) G_{5 \mathrm{X}} \\
& +B^{2} \psi^{\prime 2}\left(\frac{A^{\prime \prime} B}{2 A r^{2}}-\frac{3 A^{\prime 2} B}{2 A^{2} r^{2}}+\frac{3 A^{\prime} B^{\prime}}{2 A r^{2}}+\frac{3 A^{\prime} B \psi^{\prime \prime}}{2 A \psi^{\prime} r^{2}}-\frac{A^{\prime} B^{\prime}}{4 A B r^{2}}-\frac{A^{\prime} \psi^{\prime \prime}}{2 A \psi^{\prime} r^{2}}+\frac{A^{\prime 2}}{4 A^{2} r^{2}}\right) G_{5 \mathrm{XX}} \\
& +B^{2} \psi^{\prime}\left(\frac{A^{\prime \prime}}{A r}-\frac{5 A^{\prime 2}}{2 A^{2} r}+\frac{3 A^{\prime} B^{\prime}}{2 A B r}-\frac{A^{\prime}}{2 A r^{2}}-\frac{3 B^{\prime}}{2 B r^{2}}+\frac{3 A^{\prime}}{2 A B r^{2}}+\frac{A^{\prime} \psi^{\prime \prime}}{A \psi^{\prime} r}-\frac{\psi^{\prime \prime}}{\psi^{\prime} r^{2}}+\frac{B^{\prime}}{2 B^{2} r^{2}}+\frac{\psi^{\prime \prime}}{\psi^{\prime} B r^{2}}\right) G_{5 X \phi}-\left(\frac{B-1}{r^{2}}+\frac{B^{\prime}}{r}\right) G_{5 \phi \phi}
\end{aligned}
$$




$$
\begin{aligned}
+ & B^{2} \phi^{\prime 2}\left(\frac{2 A^{\prime}}{A r}+\frac{B^{\prime}}{B r}+\frac{2 \psi^{\prime \prime}}{\psi^{\prime} r}+\frac{1}{r^{2}}\right) G_{\mathrm{X} \phi \phi}+B^{3} \psi^{\prime 3}\left(\frac{3 A^{\prime}}{2 A r^{2}}-\frac{A^{\prime} B^{\prime}}{2 A B r}-\frac{A^{\prime} \psi^{\prime \prime}}{A \psi^{\prime} r}+\frac{B^{\prime}}{2 B r^{2}}+\frac{\psi^{\prime \prime}}{\psi^{\prime} r^{2}}+\frac{A^{\prime 2}}{2 A^{2} r}\right) G_{5 \mathrm{XX} \phi} \\
& -B^{4} \psi^{\prime 4}\left(\frac{A^{\prime} B^{\prime}}{4 A B r^{2}}+\frac{A^{\prime} \psi^{\prime \prime}}{2 A \psi^{\prime} r^{2}}-\frac{A^{\prime 2}}{4 A^{2} r^{2}}\right) G_{5 \mathrm{XXX}} .
\end{aligned}
$$

[1] C. M. Will, Living Rev. Relativity 17, 4 (2014).

[2] E. Berti et al., arXiv:1501.07274.

[3] T. Clifton, P. G. Ferreira, A. Padilla, and C. Skordis, Phys. Rep. 513, 1 (2012).

[4] D. Psaltis, Living Rev. Relativity 11, 9 (2008).

[5] N. Yunes and X. Siemens, Living Rev. Relativity 16, 9 (2013).

[6] J. R. Gair, M. Vallisneri, S. L. Larson, and J. G. Baker, Living Rev. Relativity 16, 7 (2013).

[7] G. W. Horndeski, Int. J. Theor. Phys. 10, 363 (1974).

[8] T. Damour and G. Esposito-Farese, Classical Quantum Gravity 9, 2093 (1992).

[9] M. Horbatsch, H. O. Silva, D. Gerosa, P. Pani, E. Berti, L. Gualtieri, and U. Sperhake, Classical Quantum Gravity 32, 204001 (2015).

[10] A. Padilla and V. Sivanesan, J. High Energy Phys. 04 (2013) 032.

[11] C. Charmousis, T. Kolyvaris, E. Papantonopoulos, and M. Tsoukalas, J. High Energy Phys. 07 (2014) 085.

[12] A. Nicolis, R. Rattazzi, and E. Trincherini, Phys. Rev. D 79, 064036 (2009).

[13] C. Deffayet, S. Deser, and G. Esposito-Farese, Phys. Rev. D 80, 064015 (2009).

[14] T. Kobayashi, M. Yamaguchi, and J. Yokoyama, Prog. Theor. Phys. 126, 511 (2011).

[15] C. Charmousis, Lect. Notes Phys. 892, 25 (2015).

[16] S. V. Sushkov, Phys. Rev. D 80, 103505 (2009).

[17] E. N. Saridakis and S. V. Sushkov, Phys. Rev. D 81, 083510 (2010).

[18] C. Germani and A. Kehagias, Phys. Rev. Lett. 105, 011302 (2010).

[19] C. Germani and A. Kehagias, Phys. Rev. Lett. 106, 161302 (2011).

[20] G. Gubitosi and E. V. Linder, Phys. Lett. B 703, 113 (2011).

[21] T. Kobayashi and N. Tanahashi, Prog. Theor. Exp. Phys. 2014, 73E02 (2014).

[22] C. Armendariz-Picon, V. F. Mukhanov, and P. J. Steinhardt, Phys. Rev. D 63, 103510 (2001).

[23] C. Armendariz-Picon, T. Damour, and V. F. Mukhanov, Phys. Lett. B 458, 209 (1999).

[24] M. Alishahiha, E. Silverstein, and D. Tong, Phys. Rev. D 70, 123505 (2004).

[25] C. Deffayet, G. Esposito-Farese, and A. Vikman, Phys. Rev. D 79, 084003 (2009).
[26] M. Rinaldi, Phys. Rev. D 86, 084048 (2012).

[27] M. Minamitsuji, Phys. Rev. D 89, 064017 (2014).

[28] A. Anabalon, A. Cisterna, and J. Oliva, Phys. Rev. D 89, 084050 (2014).

[29] L. Hui and A. Nicolis, Phys. Rev. Lett. 110, 241104 (2013).

[30] T. P. Sotiriou and S.-Y. Zhou, Phys. Rev. Lett. 112, 251102 (2014).

[31] T. P. Sotiriou and S.-Y. Zhou, Phys. Rev. D 90, 124063 (2014).

[32] P. Kanti, N. Mavromatos, J. Rizos, K. Tamvakis, and E. Winstanley, Phys. Rev. D 54, 5049 (1996).

[33] P. Pani and V. Cardoso, Phys. Rev. D 79, 084031 (2009).

[34] D. Ayzenberg and N. Yunes, Phys. Rev. D 90, 044066 (2014); 91, 069905 (2015).

[35] A. Maselli, P. Pani, L. Gualtieri, and V. Ferrari, Phys. Rev. D 92, 083014 (2015).

[36] B. Kleihaus, J. Kunz, and E. Radu, Phys. Rev. Lett. 106, 151104 (2011).

[37] B. Kleihaus, J. Kunz, and S. Mojica, Phys. Rev. D 90, 061501 (2014).

[38] E. Babichev and C. Charmousis, J. High Energy Phys. 08 (2014) 106.

[39] E. Babichev, C. Charmousis, and M. Hassaine, J. Cosmol. Astropart. Phys. 05 (2015) 031

[40] T. Kolyvaris, G. Koutsoumbas, E. Papantonopoulos, and G. Siopsis, Classical Quantum Gravity 29, 205011 (2012).

[41] J. B. Hartle, Astrophys. J. 150, 1005 (1967).

[42] J. B. Hartle and K. S. Thorne, Astrophys. J. 153, 807 (1968).

[43] T. Kobayashi, H. Motohashi, and T. Suyama, Phys. Rev. D 85, 084025 (2012).

[44] T. Kobayashi, H. Motohashi, and T. Suyama, Phys. Rev. D 89, 084042 (2014).

[45] A. Maselli et al. (to be published).

[46] E. Berti, V. Cardoso, and A. O. Starinets, Classical Quantum Gravity 26, 163001 (2009).

[47] M. Minamitsuji, Gen. Relativ. Gravit. 46, 1785 (2014).

[48] P. Pani, Int. J. Mod. Phys. A 28, 1340018 (2013).

[49] P. Pani, E. Berti, V. Cardoso, and J. Read, Phys. Rev. D 84, 104035 (2011).

[50] A. Cisterna, T. Delsate, and M. Rinaldi, Phys. Rev. D 92, 044050 (2015).

[51] T. Damour and G. Esposito-Farese, Phys. Rev. Lett. 70, 2220 (1993).

[52] P. Chen, T. Suyama, and J. Yokoyama, arXiv:1508.01384. 